\title{
Sentido común y conflicto: impacto de las teorías legas sobre relaciones intergrupales
}

\section{Common Sense and Conflict: Impact of Lay Theories on Intergroup Relationships}

Recibido: septiembre 14 de 2009 ～Revisado: noviembre 2 de 2009 Aceptado: noviembre 13 de 2009

\author{
LUISA RAMÍREZ ** \\ Fundación Universitaria Konrad Lorenz, Bogotá, Colombia \\ SHERY LEVY ${ }^{* * *}$ \\ Stony Brook University, New York, United States of America
}

Para citar este artículo. Ramírez, L. \& Levy, S. (2010). Sentido común y conflicto: impacto de las teorías legas sobre relaciones intergrupales. Universitas Psychologica, 9 (2), 331-343.

* Artículo de investigación.

** La correspondencia concerniente a este artículo se debe dirigir a Luisa Ramírez, quien se encuentra vinculada la Facultad de Psicología de la Fundación Universitaria Konrad Lorenz como Directora del Centro de Investigaciones, Carrera 9 bis \# 62-43. Tel: 57-1 3472311. Correo electrónico: luisaf.ramirezr@fukl.edu.co

**** Department of Psychology, 142 Psychology-B Bldg, Stony Brook, NY 11794-2500, EE.UU. Correo electrónico: sheri.levy@stonybrook.edu

\section{RESUMEN}

Teorías legas son teorías del sentido común que las personas legas utilizan para explicar y predecir fenómenos de su entorno. Evidencia empírica sugiere que las teorías legas impactan las relaciones intergrupales, entre otras razones, por su capacidad para promover tolerancia o rechazo hacia las minorías sociales. Este artículo revisa algunos hallazgos sobre la relación entre tres teorías legas (la Ética Protestante del Trabajo, la Creencia en un Mundo Justo y el Esencialismo Psicológico) y actitudes hacia miembros de grupos minoritarios en diversos contextos sociales y culturales, señalando el papel de algunas variables que inciden en su interpretación (características del perceptor y del contexto social y cultural), y sus implicaciones teóricas. Al hacerlo, establece la relevancia del estudio de varias teorías legas en el contexto latinoamericano destacando los escasos, pero sustanciales hallazgos en algunos países latinoamericanos, y llama la atención sobre la necesidad de emprender más estudios en esta dirección.

Palabras clave autores

Teorías legas, estereotipo, prejuicio, esencialismo, ética protestante del trabajo, creencia en un mundo justo.

Palabras clave descriptores

Relaciones intergrupales, estereotipo (psicología), teoría del derecho, prejuicios y antipatías.

\section{A B S T R A C T}

Lay theories are theories that lay people use to understand and predict events in their everyday life. Empirical evidence suggests that lay theories impact intergroup relations, among other things, , because of their ability to promote tolerance or rejection towards social minorities. This review focuses on research findings on the relationship between three lay theories (Protestant Work Ethic, Just World Belief and Psychological Essentialism), and attitudes toward minority groups across several social and cultural contexts, while addressing the role of other variables that may have an impact on their interpretation (perceiver and contextual characteristics). In doing so, it attempts to establish the relevance of the study of lay theories in the Latin-American context emphasizing the findings of the scarce, yet substantial, work performed so far, as well as the need to perform more research in this direction.

Key words authors

Lay theories, stereotypes, prejudice, essentialism, Protestant Work Ethic, Just, World Belief.

Key words plus

Intergroup Relations, Stereotype (Psychology), Law-Theory, Prejudices and Antipathies. 
Asumir la tarea de entender el entorno social como un ejercicio lógico-deductivo en medio de la complejidad del mismo, llevaría a cualquier persona, irrevocablemente, a una situación de inconmensurabilidad, ante la cual todos los recursos cognitivos serían insuficientes para calmar la incertidumbre que este ejercicio de procesamiento tan complejo le provocaría. Es por esto que los seres humanos nos valemos del "sentido común" para construir reglas de inferencia, que nos permiten explicar eventos relevantes en nuestro entorno social (Heider, 1958; Kelly, 1955). Tales reglas de inferencia cumplen las siguientes funciones: psicológicas puesto que ofrecen a las personas la sensación de control y certeza, lo cual a su vez mejora su autoestima; epistémicas en tanto proveen significado ayudando a las personas a entender los eventos sociales de su entorno (Heider, 1958); y, sociales en tanto contribuyen a construir y mantener el consenso social (Fletcher, 1995; Furnham, 1988; Hong et al., 2001; Levy, Chiu \& Hong, 2006; Wegener \& Petty, 1998; Yzerbyt, Judd \& Corneille, 2004). Adicionalmente, dichas reglas de inferencia permiten hacer atribuciones causales sobre los eventos del entorno y sobre el comportamiento de las personas, $y$ afectan las percepciones de responsabilidad por los resultados de una situación (Shaver \& Drown, 1986).

Trascendiendo el ejercicio individual de la búsqueda de explicaciones causales a los eventos del entorno social, es posible que tales reglas de inferencia se constituyan en teorías legas, en la medida en que entran a formar parte del "conocimiento compartido" de una sociedad. Éstas, subyacen a las dinámicas propias del sistema de relaciones sociales, y a la forma como los miembros de dicha sociedad interpretan las inequidades entre grupos socialmente relevantes (véanse también teorías legitimadoras, ideologías justificadoras, o teorías legas, Jost \& Banaji, 1994; Levy, Chiu \& Hong, 2006; Sidanius \& Pratto, 1999, respectivamente).

En este artículo, se intentará desarrollar una discusión conceptual sobre las teorías legas, destacando algunas de las variables que afectan su impacto sobre el pensamiento social. Primero, se discutirán brevemente tres teorías: la Ética Protestante del Trabajo, la Creencia en un Mundo Justo, y el Esencialismo Psicológico. Se argumentará sobre la relevancia del estudio de las teorías legas y de un enfoque social del desarrollo para la comprensión de las actitudes sociales, y el tipo de atribuciones causales y de responsabilidad que se ciernen sobre grupos sociales minoritarios, así como las consecuencias que éstas tienen en el contexto de las relaciones entre grupos socialmente relevantes en el contexto latinoamericano. Existen otros abordajes teóricos como el de las representaciones sociales que se ocupan de problemas similares, los cuales serán discutidos aquí por no ser el propósito de este artículo. Sin embargo, para una discusión sobre las relación entre el abordaje de las actitudes sociales, y el de las representaciones sociales véanse ParalesQuenza y Viscaino-Gutiérrez (2007).

\section{Teorías Legas}

Desde que Kelly (1955) y Heider (1958) orientaron sus esfuerzos al estudio de las teorías del sentido común, el estudio de estas teorías "legas" o "ingenuas" se ha convertido en un interesante foco de análisis para muchos investigadores de los procesos de percepción social (véanse, por ejemplo, Furnham, 1988; Hong et al., 2001; Wegener $\&$ Petty, 1998). La evidencia existente sugiere que las teorías legas, es decir, las teorías que la gente usa en su vida diaria para explicar eventos en su entorno social (por ejemplo, Fletcher, 1995; Furnham, 1988; Heider, 1958; Hong et al., 2001; Levy et al., 2006; Wegener \& Petty, 1998; Yzerbyt et al., 2004), sirven como marcos de referencia que influencian los procesos de percepción, interpretación de información y predicción de eventos y, en consecuencia, también las elecciones y comportamientos de las personas (véanse, por ejemplo, Furnham, 1988; Hong, Levy \& Chiu, 2001; Wegener \& Petty, 1998).

El impacto de las teorías legas (implícitas, ingenuas, intuitivas, de sentido común, o de fondo (background)) en el pensamiento social, radica precisamente en que las personas no son conscientes de la influencia que tienen sobre su manera de entender el mundo. De hecho, al no ser objeto de comprobaciones científicas, dichas teorías son 
ampliamente aceptadas y reproducidas como resultado del consenso social (Hong, Levy \& Chiu, 2001). Con frecuencia, estas teorías se expresan en refranes tales como "árbol que nace torcido su tronco nunca endereza", "a cada cual le llega lo que se merece", o "hijo de tigre sale pintado" (para un ejemplo del caso colombiano, véase CastroGómez, 2007).

Adicionalmente, estas teorías que juegan un papel central en el pensamiento social, filtrando percepciones de eventos que involucran individuos y grupos de una sociedad, dotándolos de sentido y moldeando las respuestas individuales ante tales eventos (Hong et al., 2001; Fletcher, 1995; Furnham, 1988; Levy, Chiu \& Hong, 2006; Wegener \& Petty, 1998; Yzerbyt et al., 2004), no suelen ser explícitas en sus implicaciones. Así, las teorías legas sirven no solo como marco de interpretación al procesamiento cognitivo de la información social, sino que suelen cumplir una función justificadora del statu quo, particularmente favorable para grupos o categorías sociales en condición de ventaja (Crandall, 2000). De hecho, algunos han observado que las personas que ostentan algún prejuicio pueden, de manera post hoc, crear, buscar, y adoptar teorías legas que justifican sus prejuicios (por ejemplo, Crandall, 2000; Jost \& Banaji, 1994; Kluegel \& Smith, 1986; Sidanius \& Pratto, 1994, 1999). En particular Crandall (2000), sugiere que existen por lo menos dos clases de teorías legas que cumplen esta función de justificación: aquellas que corresponden a enfoques de "atribución" (atribución de causalidad, juicios de responsabilidad y culpabilidad) y otras que corresponden a un enfoque de "jerarquización" (defensa de las jerarquías y la inequidad como necesarias, buenas, inevitables y naturales).

Teorías legas como la creencia en un mundo justo, en donde a cada quien le llega lo que merece (Crandall, 2000; Hunt, 2000), la Ética Protestante del Trabajo según la cual cualquier persona, mediante el trabajo duro, puede ayudarse a sí misma a salir de una mala situación y conseguir el éxito (Furhnam, 1988; Levy, West, Ramírez \& Karafantis, 2006) corresponderían al primer grupo (atribución). Otras como la Orientación a la Dominancia
Social, o creencia en que las jerarquías sociales son buenas y necesarias (Sidanius \& Pratto, 1999); las versiones modernas del Darwinismo Social que utilizan argumentos pseudocientíficos basados en hallazgos en el campo de la genética para explicar las diferencias "visibles" entre diferentes categorías sociales (Crandall, 2000), o la teoría de la "ceguera al color" (Color-Blind Theory), según la cual, la información sobre las categorías sociales (como la raza o el género) es irrelevante (Levy, West \& Ramirez, 2005), corresponderían al segundo grupo (jerarquización). Por último, existen también teorías legas que combinan argumentos de atribución y jerarquización en la justificación del prejuicio social (Crandall, 2000). Más adelante se argumentará que la teoría lega del esencialismo psicológico, que explica las diferencias entre categorías sociales con argumentos de determinismo biológico o social (Haslam et al., 2000, 2002; Jayaratne et al., 2006; Keller, 2005; Mahalingham, 2003), pertenece a este grupo.

\section{Teorías legas de tipo atributivo}

\section{Ética [Protestante] del Trabajo}

La teoría lega de la Ética Protestante del Trabajo (EPT) ha recibido atención de muchos investigadores a lo largo y ancho del mundo desde hace varias décadas (véanse, por ejemplo, los trabajos de Furhnam y colaboradores en Barbados, Inglaterra, Malasya, Japón, y otros). Se trata de una teoría lega que enfatiza el trabajo duro, la conservación de recursos y la evitación de la haraganería (Zubieta, 2005). La EPT es una teoría de un amplio dominio conceptual cuyas implicaciones impactan dominios abstractos como las actitudes políticas: el conservatismo, el autoritarismo o las actitudes frente a la inequidad (Levy, Freitas \& Salovey, 2002). Adicionalmente, la EPT impacta dominios muy específicos como las actitudes frente al trabajo (Furhnam, 1991; Furnham \& Reilly, 1991; Niles, 1999), el dinero (Furnham, 1987), o las atribuciones de responsabilidad sobre la apariencia personal (Crandall \& Martínez, 1996).

Gran número de estudios conducidos en diferentes partes del mundo sobre la ética protestante 
del trabajo, sugieren que ésta se relaciona con prejuicio social y oposición a los esfuerzos igualitaristas de algunos grupos sociales (Levy et al., 2005; Zubieta, 2005). Así pues la EPT se relaciona con actitudes negativas hacia las personas en condición de mendicidad o sin techo (Sommerman, 1993), personas con sobrepeso (Biernat, Vescio, Theno \& Crandall, 1996; Crandall, 1994, 2000; Crandall \& Martínez, 1996), miembros de grupos étnicos o raciales (Katz \& Hass, 1988; McConahay \& Hough, 1976), Gente Gay (Biernat et al., 1996), y personas infectadas con el virus del SIDA (Crandall \& Coleman, 1992; Levy, Freitas \& Salovey, 2002) entre otros. Adicionalmente, la EPT ha sido relacionada con la tendencia a culpar a las personas por la pobreza o el desempleo (Heaven, 1990). En consecuencia, muchos investigadores han esperado encontrar una correlación positiva entre la EPT y el prejuicio social.

No obstante, la evidencia empírica encontrada es controversial. De hecho algunos hallazgos sugieren que la relación entre la EPT y el prejuicio social puede estar moderada por la saliencia de otros sistemas de valores como, por ejemplo, teorías legas igualitaristas (Biernat, Vescio \& Theno, 1996), o bien que es posible interpretar teorías igualitaristas de manera que resultan compatibles con la EPT. En consecuencia, ésta última parece relacionarse con actitudes de tolerancia hacia ciertos grupos sociales (Monteith \& Walters, 1998, estudio piloto).

Levy, West, Ramírez y Karafantis (2006) postularon que la Ética Protestante del Trabajo podía adquirir por lo menos dos significados con diferentes implicaciones en términos de las relaciones entre grupos. Por un lado, la EPT tendría un significado igualitario, puesto que la creencia en que todo aquel que trabaja duro logra el éxito implicaría que a la base, todos somos iguales. Por otro, la EPT tendría un significado mucho menos igualitario. Para dar un ejemplo, la teoría de que las personas que trabajan duro tienen éxito puede ser utilizada para adelantar el argumento de que aquellos que no tienen éxito no trabajan lo suficientemente duro (atribución causal), o sugerir que la falta de éxito es consecuencia de la pereza o de algún otro determinante que descarga la culpa de la adversidad en las personas y no en sus circunstancias (atribución de responsabilidad).

Levy et al. (2006) plantearon que las personas acumulan y refinan su comprensión de las teorías legas a través de sus experiencias en contextos sociales y culturales y, por lo tanto, sus implicaciones pueden variar en dichos contextos. Dicho de otra forma, una razón por la cual una teoría lega puede tener más de un significado o implicación para las relaciones entre grupos, consiste en que ésta puede tener un significado de base, y otro asociado como resultado de la experiencia en un contexto social y cultural determinado. Así pues, la exposición a situaciones culturalmente relevantes que asocian el uso de la EPT a situaciones que expresan intolerancia social hacia los miembros de ciertas minorías sociales, debería conducir a que aquellas personas con mayor experiencia expresen con más frecuencia las implicaciones intolerantes de la EPT.

En un estudio de tipo correlacional entre estudiantes de tres grupos de edad pertenecientes a escuelas públicas en Estados Unidos y en Colombia, Levy et al. (2006) encontraron evidencia de que, con la experiencia, en Estados Unidos, la relación entre la EPT y el prejuicio social se fortalece con la edad, mientras que en Colombia la asociación entre la EPT y el prejuicio social permanece relativamente estable a lo largo del proceso de socialización. Esta discrepancia entre los hallazgos en Colombia y en Estados Unidos apoya la intuición de estas investigadoras en cuanto a la dualidad en los significados de la Ética Protestante del Trabajo y el papel de los procesos de socialización en diferentes contextos. Esto no significa que en Colombia no ocurra la asociación entre la EPT y el prejuicio social; solo que dicha asociación no parece formar parte de una postura ideológica organizada, y no suele ser objeto explícito de discusión.

De hecho, otras investigaciones realizadas en contextos más similares al colombiano ofrecen evidencia en favor de esta intuición. Crandall y Martinez (1996) intuyeron que las actitudes de prejuicio en contra de personas con sobrepeso, se relacionan con teorías legas que tienden a atribuir la responsabilidad de su situación a los individuos y no a otros posibles determinantes de índole social, 
cultural, económica. Entonces, es posible que las actitudes frente a los miembros de ciertas minorías sean explicadas por las atribuciones acerca de la controlabilidad que tienen los individuos sobre su situación. Consistentemente, los autores encontraron evidencia a favor de la relación entre la EPT y actitudes de intolerancia frente a las personas con sobrepeso. No obstante, una comparación transcultural permitió establecer que esta asociación era mucho más fuerte entre miembros de la cultura norteamericana que entre los de la mexicana. Es decir, como en el caso colombiano, la asociación entre EPT e intolerancia ocurrió, mas no con la misma fuerza que en la cultura norteamericana.

Por otra parte, evidencia recogida recientemente en Argentina apoya la intuición de la importancia del proceso de socialización en el establecimiento de la relación ente la EPT y ciertas actitudes sociales. Zubieta (2005) comparó las creencias en la EPT y la competitividad entre 225 estudiantes universitarios de Ciencias Económicas y de Psicología de la ciudad de Buenos Aires, para indagar si existe una asociación entre la carrera de estudios y teorías legas sobre el entorno. En efecto, Zubieta encontró diferencias significativas entre los estudiantes de Ciencias Económicas y Psicología, en lo que concierne a la creencia en la EPT y la competitividad. Los estudiantes de ciencias económicas reportaron valores mucho más altos en estas escalas que los de Psicología. Además de la importancia de esta teoría lega entre la población latinoamericana (a pesar de una tradición religiosa que se distancia de la EPT), la investigación de Zubieta sugiere también que distintos contextos de socialización (Ciencias Económicas o Psicología) se asocian de manera diferente con la adopción de estas teorías legas, lo cual es consistente con las afirmaciones de Levy et al. (2005), y respecto a la variabilidad de las implicaciones de la EPT en diferentes contextos de socialización.

\section{Creencia en un Mundo Justo}

Lerner (1970) argumentó que las personas tienen la necesidad de creer que obtienen lo que merecen. Este sistema de creencias constituye una teoría lega conocida como la Creencia en un Mundo Justo (CMJ). Evidencia empírica a favor de esta intuición sugiere que por una parte la CMJ permite que las personas confíen en recibir un trato justo, y que así mismo traten de manera justa a los demás (Correia \& Dalbert, 2008). Las autoras exploraron la relación entre la CMJ y el matoneo o acoso escolar en 197 jóvenes adolescentes de séptimo y noveno grado, en una escuela en Portugal. Argumentaron que en la medida en que la CMJ cumple una función de motivación (Lerner, 1980), ésta adquiere un papel fundamental en la explicación del comportamiento agresivo, tal que debe estar negativamente asociada con comportamientos injustos que rompen el contrato personal. Conforme a sus expectativas, las autoras encontraron que aquellos estudiantes que defienden la CMJ tienen menor probabilidad de involucrarse en acoso escolar hacia sus compañeros. Esto sugiere que solo en la medida en que se actúa con justicia, pueden las personas tener la expectativa de que otros los traten de la misma forma.

No obstante, otra evidencia sugiere que la CMJ promueve la asimilación de injusticias que incluyen desde la atribución de responsabilidad por situaciones que están fuera del control personas (Crandall \& Martínez, 1996), como el estupro (Herbert \& Dunkel-Schetter, 1992), hasta la justificación de las horrendas acciones cometidas por dictadores en diversos contextos (IJzerman \& Prooijen, 2008). De hecho, Herbert y Dunkel-Schetter (1992) argumentaron que la CMJ constituye uno de los factores que median la respuesta de los miembros de una red social hacia una persona que ha sido victimizada.

Crandall y Martinez (1996, citados arriba) también encontraron evidencia a favor de la asociación entre la CMJ y el rechazo hacia personas con sobrepeso. Es decir, la creencia en la controlabilidad de un evento (es decir la atribución de responsabilidad sobre el sobrepeso) aparece asociada con actitudes negativas hacia los miembros de dicha categoría.

IJzerman y Prooijen (2008) sugieren que la creencia en un mundo justo se relaciona con la dificultad para regular el afecto negativo. Frente a un evento incongruente con la CMJ, algunas 
personas tienden a la cavilación de ideas lo cual les permite reducir dicha incongruencia entre su sistema de creencias y la situación que lo cuestiona. Como consecuencia, este tipo de personas podrían, por ejemplo, apoyar políticos de línea dura incluso tras cometer crímenes horrendos (IJzerman \& Prooijen, 2008). No es de sorprenderse entonces que varios hallazgos de investigación hayan relacionado la creencia en un mundo justo con la tendencia a culpar a la víctima, incluso en situaciones que parecen evidentemente una victimización. La $\mathrm{CMJ}$ subyace a argumentos que se citan con frecuencia en el discurso diario para tratar de explicar acciones criminales como los asesinatos (como cuando las personas explican: imurió porque era un criminal!, o, iAlgo debería!) (IJzerman \& Prooijen, 2008), el estupro (como cuando las personas atribuyen la responsabilidad de la violación a la víctima argumentando que ésta es responsable por algún aspecto de su comportamiento como su vestimenta) (Crandall, 2000; Wyer, Bodenhausen \& Gorman, 1985).

\section{Esencialismo Psicológico}

El esencialismo psicológico representa una teoría lega sobre de las diferencias entre los grupos sociales (véanse Estrada, Yzerbyt \& Seron, 2004; Gottfriend \& Gelman, 2005; Yzerbyt, Rogier \& Fiske, 1998). Se trata de una heurística que indica que ciertos grupos poseen $\mathrm{X}$ características (por ejemplo, son inteligentes) mientras que otros grupos poseen Y características (por ejemplo, son sociables). El término esencialismo psicológico fue propuesto inicialmente por Medin (1989) y Medin y Orthony (1989) en su teoría sobre los procesos de categorización. Los autores propusieron que durante el proceso de categorización las personas trabajan bajo el supuesto de que las cosas que parecen similares comparten, por lo general, cualidades profundas que son esenciales a su pertenencia a una categoría común. Para dar un ejemplo, una silla puede seguir siendo una silla sin importar el número de patas, la forma de asiento, etc., gracias a aspectos esenciales que la clasifican en dicha categoría. Algunos estudiosos del tema sostienen que esta heurística que concibe las características superficiales de los objetos y de las personas como una expresión de su naturaleza subyacente es parte fundamental de nuestro sistema cognitivo, puesto que, con frecuencia, constituye una manera efectiva de pensar nuestro mundo social desde muy temprana edad (véanse Gelman 2003; Rangel \& Keller, en prensa). Sin embargo, algunos autores han destacado las consecuencias menos deseables del esencialismo en el sentido de que las diferencias entre grupos son naturales y por lo tanto las inequidades entre los mismos son inevitables (Gelman, 2003; Haslam et al., 2000, 2002; Hirschfeld, 2001).

Rothbart y Taylor (1992) propusieron que el pensamiento esencialista involucra un mal entendimiento de grupos construidos socialmente como si se tratara de clases o categorías "naturales", es decir, algo similar a una especie biológica. Por ejemplo, en la medida en que la gente cree que la raza o el origen étnico representan algo similar a una "clase natural", el color de la piel se convierte en un atributo que representa la esencia del grupo. En consecuencia, la gente tiende a asumir que los miembros de un grupo racial comparten entre sî cualidades profundas que los hacen diferentes a los miembros de otros grupos. De manera tácita, se asume que esta esencia determina la identidad de los miembros de este grupo y los hace a todos parecidos en "lo fundamental". Keller y Bless (2004) señalan que, en la teoría clásica evolutiva, las diferencias individuales en características fenotípicas reflejan ventajas reproductivas heredables por vía genética. Así como la teoría evolutiva supone la existencia de predisposiciones genéticas basadas en adaptaciones heredadas genéticamente, la creencia en tales predisposiciones refleja una forma de esencialismo psicológico. Éste, a su vez, se refleja en las teorías legas que la gente utiliza para construir representaciones mentales de los grupos sociales, y explicar y justificar el mundo social que los rodea.

En este sentido, el esencialismo por su efecto "naturalizador" sobre las diferencias intergrupales, se convierte en una teoría lega que defiende y justifica las jerarquías sociales. Es decir, una teoría lega jerarquizante para ponerlo en los términos de Crandall (2000). No obstante, esta aproximación 
que destaca el papel de la construcción de explicaciones que enfatizan las comunalidades (esencias) biológicas entre los miembros de una categoría, parece ser solo una parte de la historia.

Estudios más recientes sugieren que el pensamiento esencialista se ordena alrededor de dos dimensiones que denotan dos tipos de argumentos deterministas (biológico y social) tradicionalmente conocidas como la dimensión de Clases $\mathrm{Na}$ turales y de Entitatividad (por ejemplo, Bastian \& Haslam, 2006; Demoulin, Leyens \& Yzerbyt, 2006; Haslam et al., 2000, 2002). La dimensión de Clases Naturales involucra la creencia en que las categorías sociales son immutables, es decir que la membresía en un grupo es permanente y sus miembros no pueden cambiar su identidad (por ejemplo, una persona que ha sido gay siempre lo será aunque intente cambiarlo); discretas es decir, la membresía en estas categorías es cuestión de todo o nada (se es hombre o se es mujer pero no es posible estar en la mitad); históricamente invariables, es decir independientes del leguaje y la cultura. Adicionalmente, tienen características que las definen como necesarias, suficientes y naturales, lo cual implica una base cuasibiológica (Haslam et al., 2002; Hirschfeld, 1993; Rothbart \& Taylor, 1992; Yzerbyt, Corneille \& Estrada, 2001; Yzerbyt, Rogier \& Fiske, 1998).

La otra dimensión del pensamiento esencialista, entitatividad, tiene que ver con la percepción del grupo como un ente (por ejemplo, Brewer \& Harasty, 1996; Campbell, 1958; Hamilton \& Sherman, 1996; Yzerbyt et al., 2004). Este componente involucra la creencia en que las categorías sociales poseen características que les son inherentes, como un tipo de esencia subyacente que se expresa en las características superficiales del grupo; uniformes $u$ homogéneas, informativas acerca de sus miembros, y determinan su identidad (Haslam et al., 2002). Algunos ejemplos de categorías sociales que son percibidas como entitativas son los grupos políticos y religiosos (judíos, por ejemplo), las personas gay, los pacientes con SIDA y los grupos étnicos (Haslam et al., 2000).

Más recientemente Rangel y Keller (en prensa) distinguen entre la creencia en el determinismo genético y la creencia en el determinismo social, la cual implica que las características esenciales de las personas (reflejadas en su "carácter social") son moldeadas por su ambiente social (crianza, socialización, bagaje social, etc.). Esta creencia correlaciona positivamente con la tendencia a percibir homogeneidad en los grupos sociales (Rangel \& Keller, en prensa). Adicionalmente, al igual que la creencia en la determinación genética y otras creencias que forman parte de la teoría lega del esencialismo psicológico, la creencia en el determinismo social correlaciona con ciertas necesidades epistémicas como el dogmatismo, la baja tolerancia a la ambigüedad, y con el prejuicio y la discriminación social (véanse Keller, 2005; Rangel $\&$ Keller, en prensa).

Muchos autores han destacado en sus perspectivas el papel de la teoría lega del esencialismo psicológico como un argumento justificador de la inequidad entre grupos o categorías sociales (Demoulin, Leyens \& Yzerbyt, 2006; Gelman \& Taylor, 2000; Haslam, Bastian et al., 2006; Haslam et al., 2000; Mahalingam, 2003; Mahalingam \& Leu, 2005, para dar algunos ejemplos), entre otras razones por las atribuciones causales ancladas a discursos esencialistas sobre los mismos (Haslam et al., 2000, 2002; Jayaratne et al., 2006; para dar solo algunos ejemplos).

En síntesis, por su efecto naturalizador, el esencialismo psicológico cumpliría una función jerarquizante según la definición de Crandall (2000), puesto que refuerza y justifica la creencia en la naturalidad de las diferencias intergrupales. No obstante, como destacan Rangel y Keller, las dos dimensiones del pensamiento esencialista implican a su vez atribuciones causales acerca de aquellos aspectos que son propios de la pertenencia a una categoría social, y que determinan la identidad de los miembros del grupo, dando lugar a argumentos de determinismo biológico o social.

\section{Esencialismo y prejuicio social}

Las creencias esencialistas se han visto relacionadas con mayores niveles de prejuicio hacia grupos étnicos minoritarios en los Estados Unidos, 
Alemania, los Países Bajos y España (Jayaratne et al., 2006; Keller, 2005; Levy et al., 2001; Leyens, Rodríguez-Pérez, Rodríguez-Torres, Gaunt, Paladino, Vaes \& Demoulin, 2001; y, Verkuyten \& Brug, 2004) contra las mujeres en Estados Unidos (Haslam, Rothschild et al., 2002) y en la India (Mahalingam, 2003), contra los transgeneristas en Inglaterra (Tee \& Hegarty, 2006), y contra las personas provenientes de castas inferiores (Dahlits) en la India (Mahalingam, 2003), entre otros. Esto ha llevado a varios investigadores a argumentar que la teorías esencialistas sobre la naturaleza de los grupos ayudan a justificar relaciones inequitativas dentro de la sociedad, en tanto ofrecen explicaciones que parecen sensatas a un observador desprevenido sobre las inequidades existentes entre grupos dominantes y minoritarios de la sociedad (como grupos étnicos, raciales, mujeres, discapacitados, inmigrantes, y otros), (Demoulin et al., 2000; Haslam et al., 2006; Haslam et al., 2000; Mahalingam, 2003; Mahalingam \& Leu, 2005). No obstante, las creencias esencialistas también se han visto relacionadas con menores niveles de prejuicio contra las personas gay en Estados Unidos e Inglaterra (Hegarty, 2002; Hegarty \& Parto, 2001; Jayaratne et al., 2006).

Existe evidencia a favor de la perspectiva del desarrollo social de Levy y colaboradores (2005, 2006), que sugiere que como otras teorías legas, el esencialismo psicológico, puede ser activado a través de manipulaciones experimentales (Keller, 2005; Levy et al., 1998; Rangel \& Keller, en prensa; Williams \& Eberhardt, 2008; por mencionar algunos). La implicación de estos hallazgos es que el procesamiento de información sobre las categorías sociales es afectado por el grado de exposición en el contexto social a argumentos esencialistas sobre las mismas. Para dar algunos ejemplos Levy et al. (1998) obtuvieron evidencia de una relación causal entre la exposición a la creencia en la estabilidad de los rasgos (Teorías Implícitas) de un grupo racial y el estereotipamiento de estos grupos por parte de un grupo mayoritario. Aunque, tradicionalmente, las Teorías Implícitas de la Personalidad han sido estudiadas de manera independiente, (véanse, por ejemplo, Caridad, Cepero \& McCoach, 2008;
Levy et al., 1998) existe evidencia que sugiere una fuerte relación entre dichas teorías y la creencia esencialista en la inmutabilidad de las categorías sociales. Por ejemplo, los resultados de Levy et al. (1998) fueron replicados más tarde por Bastian y Haslam (2006), quienes obtuvieron evidencia de la relación entre la exposición a varias creencias esencialistas (las teorías implícitas de la personalidad, inmutabilidad, la creencia en una base biológica, la informatividad y la discreción) y el pensamiento estereotipado acerca de varias categorías sociales (japoneses, aborígenes y judíos).

Otras fuentes de evidencia sobre el esencialismo psicológico sugieren también que su efecto de la exposición y la activación sobre el prejuicio social es más fuerte entre quienes de manera crónica aceptan y/o defienden argumentos esencialistas a cerca de las categorías sociales. Keller (2005) y Rangel y Keller (en prensa) realizaron una serie de estudios en los que midieron la creencia en la determinación genética de los grupos (Keller, 2005) y la determinación social de los grupos (Rangel \& Keller, en prensa), y encontraron evidencia de que cada una de estas creencias contribuye de manera independiente a predecir el uso de estereotipos negativos y prejuicio en contra de los inmigrantes de Europa del Este en el primer estudio, y de los Turcos en Alemania en el segundo, entre miembros del grupo mayoritario (europeos blancos). Adicionalmente, encontraron que ambas creencias se relacionan positiva y significativamente con la tendencia a defender la inequidad y las jerarquías sociales (conservatismo, orientación a la dominación social, patriotismo, nacionalismo).

En otro momento, utilizando la misma metodología en ambos estudios, Keller (2005) y Rangel y Keller (en prensa) activaron creencias de determinismo genético y social a un grupo de estudiantes universitarios. Los hallazgos de estos estudios ofrecen evidencia en favor de la relación causal entre la activación del determinismo genético y del determinismo social, y la tendencia a generar actitudes más positivas hacia ciudadanos de Europa occidental que de Europa del Este (tendencia a favorecer al endogrupo). Este efecto fue más fuerte entre quienes crónicamente defienden argumentos 
de determinismo social, lo cual argumenta a favor del uso funcional del esencialismo.

Así mismo, recientemente Williams y Eberhardt (2008) encontraron evidencia de que la exposición a información consistente con una concepción biológica de la "raza" podría servir como un detonante activador de la noción de pertenencia (affiliation) a un grupo, de tal manera que las personas que tienden a creer de manera crónica en este argumento tenderán a querer afiliarse con otras personas dentro de su grupo biológico. Nuevamente, estos hallazgos fueron más fuertes entre quienes ya parecen, a lo mínimo, aceptar la creencia esencialista con base biológica de las diferencias raciales.

Consistentemente con la intuición acerca del uso funcional del esencialismo psicológico, Halley (1994 citado por Hegarty \& Pratto, 2001) sugirió que las creencias en la base biológica de las diferencias intergrupales pueden causar actitudes, pero que también las actitudes pueden afectar la asimilación e interpretación de este tipo de argumentos acerca de las categorías sociales. Para evaluar esta hipótesis Hegarty y Pratto (2001) manipularon la exposición a argumentos esencialistas, pidiendo a los participantes que leyeran un artículo que contenía un reporte falso sobre "orden de nacimiento y orientación sexual", y encontraron que la creencia en la inmutabilidad, más no la creencia en el carácter discreto de la orientación sexual afectaba la asimilación (entendida como aprobación o desaprobación) de argumentos de determinismo biológico. Los autores interpretaron este hallazgo como posible evidencia de que la relación entre la creencia en el carácter discreto de la categoría y las actitudes hacia la gente gay, pueden ser el resultado de la motivación de las personas para diferenciar entre los grupos, para poder discriminar a favor del propio, de acuerdo con los principios de la teoría de la identidad social (Tajfel, 1984).

Otro ejemplo de la dualidad de las implicaciones esencialistas, así como del uso funcional de las mismas es ofrecido por Mahalingam y Rodríguez (2003), en la India. Los autores indagaron sobre la percepción de fluidez (es decir, la capacidad de cambiar o no la identidad de género) de la iden- tidad de hombres y mujeres, y encontraron diferencias significativas, sugiriendo que la identidad femenina tiende a ser percibida como más estable (menos fluida) que la identidad masculina.

Los resultados de Mahalingham y Rodríguez (2003), son consistentes con los hallazgos de Glick et al. (2000), acerca de la existencia de una correlación positiva entre el prejuicio benevolente y el prejuicio hostil hacia las mujeres, y los de Eagly y Mladinic (1994), que destacan la convivencia entre el estereotipamiento positivo de la mujer y las relaciones inequitativas entre los géneros. $\mathrm{Al}$ parecer, entonces, el estereotipamiento positivo de las mujeres, en lo que los autores denominan "culturas de honor", es consistente con las actitudes negativas hacia las transgresiones de rol, cuando no con expresiones abiertas de hostilidad hacia las mismas. Se trata de un claro ejemplo de una situación en la que creencias deterministas de tipo biológico o la naturalización de una categoría social, tienen un efecto inmovilizador que mantiene la jerarquía entre los grupos.

Por último, existe evidencia de que grupos étnicos minoritarios en todo el mundo utilizan argumentos esencialistas basados en argumentos de determinismo social, para defender el derecho de su gente a mantener su identidad cultural en un contexto multicultural (Verkuyten \& Brug, 2004). Para dar un ejemplo más cercano del primer caso, la Constitución colombiana de 1991, en la cual participaron los grupos étnicos indígenas y afroamericanos, eliminó el asimilacionismo (postura ideológica construida sobre argumentos de carácter biológico) en favor del multiculturalismo, cuyos argumentos se construyen sobre nociones más cercanas al determinismo social (Arocha, 1998). Es decir, es posible que el estatus del grupo modere el uso y el tipo de de argumentos esencialistas que se tejen sobre los grupos, y sus efectos sobre el prejuicio social (Verkuyten, 2003).

\section{Conclusión}

En síntesis, la dualidad en las implicaciones del varias teorías legas, sugiere que el impacto de dichas teorías puede ser moderado por las características 
del perceptor social, tales como el grupo de edad y momento del ciclo vital por el que atraviesa, el género, el nivel socioeconómico (Levy, West \& Ramírez, 2005; Levy, West, Ramírez \& Karafantis, 2006; véase, también, Levy, Chiu et al., 2006), la orientación política y la identificación religiosa (Jayaratne et al., 2006; Keller, 2005; Rangel \& Keller, en prensa), entre otras. Complementariamente, las implicaciones de dichas teorías legas también varían dependiendo de características del contexto social inmediato tales como el estatus relativo entre ambos grupos (Verkuyten, 2003) y del contexto cultural. Basados en estas investigaciones, Levy et al. $(2005,2006)$ sostienen, que algunas teorías legas son buenas candidatas para tener implicaciones duales y para ser utilizadas de diferentes maneras por diferentes personas $y / 0$ por la misma persona en diferentes contextos, en ocasiones llevando a mayores niveles de tolerancia y en ocasiones a mayores niveles de prejuicio contra grupos minoritarios, por varias razones. Primero, estas teorías pueden justificar el trato desigual a miembros de diferentes grupos sociales elevando argumentos aparentemente igualitarios, sugiriendo que la desigualdad social es una respuesta adecuada. Segundo, están vaga y ampliamente definidas, lo cual permite variabilidad en su uso y sus implicaciones. Tercero, en la medida en que las personas se encuentran permeadas por estas teorías culturalmente aceptadas, encuentran difícil rechazarlas a pesar de sus implicaciones.

El estudio del las teorías legas, reviste importancia no solo su contribución a la comprensión de los procesos de categorización social, sino por sus implicaciones en las relaciones intergrupales, y en particular por su capacidad para promover tolerancia o rechazo hacia las minorías sociales. No obstante, tales procesos sociales no han recibido suficiente atención desde esta perspectiva de la Psicología Social en el contexto latinoamericano. Este artículo, establece la relevancia del estudio de varias teorías legas, discutiendo los aportes de estudios anteriores en la comprensión de las relaciones entre grupos culturalmente relevantes en varios contextos. Adicionalmente, pone de relieve el papel de otras variables tales como característi- cas del perceptor, del contexto inmediato, y de la cultura, en la explicación de las particularidades de cada caso.

\section{Referencias}

Arocha, J. (1998). Inclusion of Afro-Colombians: Unreachable national goal? Latin American Perspectives, 25, 70-89.

Bastian, B. \& Haslam, N. (2006). Psychological essentialism and stereotype endorsement. Journal of Experimental Social Psychology, 42, 228-235.

Biernat, M., Vescio, T. K. \& Theno, S. A. (1996). Violating American values: A"value congruence" approach to understanding outgroup attitudes. Journal of Experimental Social Psychology, 32, 387-410.

Brewer, M. B. \& Harasty, A. S. (1996). Seeing groups as entities: The role of perceiver motivation. In Richard M. Sorrentino \& E. Tory Higgins (Eds.), Handbook of motivation and cognition (Vol. 3, pp. 347-370). New York: Guilford Press.

Campbell, D. T. (1958). Common fate, similarity and other indices of the status of aggregates of persons as social entities. Behavioral Sciences, 3, 14-25.

Castro-Gómez, S. (2007). Razas que decaen, cuerpos que producen. Una lectura del campo intelectual colombiano (1904-1934). En Rubén A. Sánchez (Ed.), Biopolítica y formas de vida (pp. 197-142). Bogotá: Editorial Pontificia Universidad Javeriana.

Correia, I. \& Dalbert, C. (1998/2008). School bullying: Belief in a personal just world of bullies, victims, and defenders. European Psychologist, 13(4), 248 254.

Crandall, C. (2000). Ideology and lay theories of stigma: The justification of stigmatization. In T. Heatherton, R. Kleck, M. Hebl \& J. Hull (Eds.), The Social Psychology of Stigma (pp. 126-150). New York: The Guildford Press.

Crandall, C. S. (1994). Prejudice against fat people: Ideology and self-interest. Journal of Personality and Social Psychology, 66, 882-894.

Crandall, C. \& Coleman, R. (1992). AIDS-related stigmatization and the disruption of social relationships. Journal of Social and Personal Relationships, 9, 163-177. 
Crandall, C. S. \& Martínez, R. (1996). Culture, ideology, and antifat attitudes. Personality and Social Psychology Bulletin, 22, 1165-1176.

Demoulin, S., Leyens J-P. \& Yzerbyt, V. (2006). Lay theories of essentialism. Group Processes \& Intergroup Relations, 9, 25-42.

Eagly, A. H. \& Mladinic, A. (1994). Are people prejudiced against women? Some answers from research on attitudes, gender stereotypes and judgments of competence. In W. Stroebe \& M. Hewstone (Eds.), European Review of Social Psychology (Vol. 5, pp. 1-35). New York:Wiley.

Estrada, C., Yzerbyt, V. \& Seron, E. (2004). Efecto del esencialismo psicológico sobre las teorías ingenuas de las diferencias intergrupales. Psichotema, 16, 181-186.

Feldman, S. (1988). Structure and consistency in public opinion: The role of core beliefs and values. American Journal of Political Science, 32, 416-440.

Fletcher, G. (1995). The scientific credibility of folk psychology. Mahwah, NJ: Lawrence Erlbaum Associates.

Furnham, A. (1988). Lay theories: Everyday understandings of problems in the social sciences. New York: Pergamon Press.

Furnham, A. (1991). The protestant work ethic in Barbados. Journal of Social Psychology, 131, 29-44.

Furnhan, A. \& Muhiudeen, C. (1984). The protestant work ethic in Britain and Malasya. The Journal of Social Psychology, 122, 157-161.

Furnham, A. \& Reilly, M. (1991). A cross cultural comparison of British and Japanese protestant work ethic and just world beliefs. Psychologia, 34, 1-14.

García-Cepero, M. C. \& McCoach, B. (2008). Educators' implicit theories of intelligence and beliefs about the identification of gifted students. Universitas Psychologica, 8(2), 295-310.

Gelman, S. A. (2003). The essential child: Origins of essentialism in everyday thought. London: Oxford University Press.

Glick, P., Fiske, S. T., Mladinic, A., Saiz, J. L., Abrams, D., Masser, B. et al. (2000). Beyond prejudice as simple antipathy: Hostile and benevolent sexism across cultures. Journal of Personality and Social Psychology, 79(5), 763-775.

Gottfried, G. \& Gelman, S. (2005). Developing domainspecific causal explanatory frameworks: The role of insides and immanenece. Cognitive Development, 20, 137-158.

Hamilton, D. L. \& Sherman, S. J. (1996). Perceiving persons and groups. Psychological Review, 103(2), 336-355.

Haslam, N., Bastian, B., Bain, P. \& Kashima, Y. (2006). Psychological essentialism, implicit theories and intergroup relations. Group Processes and Intergroup Relations, 9, 63-76.

Haslam, N. \& Levy, S. R. (2006). Essentialist beliefs about homosexuality: Structure and implications for prejudice. Personality and Social Psychology Bulletin, 32, 471-485.

Haslam, N., Rothschild, L. \& Ernst, D. (2000). Essentialist beliefs about social categories. British Journal of Social Psychology, 39, 113-127.

Haslam, N., Rothschild, L. \& Ernst, D. (2002). Are essentialist beliefs associated with prejudice? British Journal of Social Psychology, 41, 87-100.

Heaven, P. C. (1990). Human values and suggestions for reducing unemployment. British Journal of Social Psychology, 29, 257-264.

Heider, F. (1958). The psychology of interpersonal relations. New York: Wiley.

Herbert, B. \& Dunkel-Schetter, C. (1992). Negative social reactions to victims: An overview of responses and their determinants. In L. Montada, S. H. Filipp \& M. J. Lerner (Eds.), Life Crises and Experiences of Loss in Adulthood (pp. 497-518). Hillsdale, NJ: Lawrence Erlbaum.

Hegarty, P. (2002). 'It's not a choice, it's the way we're built': Symbolic beliefs about sexual orientation in the U.S. and Britain. Journal of Community and Applied Social Psychology, 12, 153-166.

Hegarty, P. \& Pratto, F. (2001). The effects of social category norms and stereotypes on explanations for intergroup differences. Journal of Personality and Social Psychology, 80, 723-735.

Hegarty, P. \& Pratto, F. (2001). Sexual orientation beliefs: Their relationship to anti-gay attitudes and biological determinist arguments. Journal of Homosexuality, 41, 121-135.

Hirschfeld, L. (1993). Do children have a theory of race? Cognition, 54, 209-252.

Hirschfeld, L. (1994). La adquisición de categorías sociales se basa en una competencia dominio- 
específica o en la transferencia de conocimientos? En L. Hirschfeld \& S. Gelman (Eds.), Cartografía de la mente (pp. 285-329). Gedisa: Barcelona.

Hirschfeld, L. (2001). On a folk theory of society: Children, evolution and mental represenations of social groups. Personality and Social Psychology Review, 5, 107-117.

Hong, Y. Y., Levy, S. R. \& Chiu, C. Y. (2001). The contribution of the lay theories approach to the study of groups. Personality and Social Psychology Review, 5, 98-106.

Hunt, M. O. (2000). Status, religion, and the "belief in a just world": Comparing African Americans, Latinos and Whites. Social Science Quarterly, 81, 325-343.

IJzerman, H. \& Van Prooijen, J-W. (2008). Just world and the emotional defense of self. Social Psychology, 39(2), 117-120.

Jayaratne, T., Ybarra, O., Sheldon, J., Browm, T., Feldbaum, M., Pfeffer, C. et al. (2006). White American's genetic lay theories of race differences and sexual orientation: Their relationship with prejudice toward black, and gay men and lesbians. Group Processes $\mathcal{E}$ Intergroup Relations, 1, 77-94.

Jost, J. \& Banaji, M. (1994). The role of stereotyping in system-justification and the production of false consciousness. British Journal of Social Psychology, 33, 1-27.

Katz, I. \& Hass, R. G. (1988). Racial ambivalence and American value conflict: Correlational and priming studies of dual cognitive structures. Journal of Personality and Social Psychology, 55, 893-905.

Keller, J. (2005). In genes we trust: The biological component of psychological essentialism and its relationship to mechanisms of motivated social cognition. Journal of Personality and Social Psycho$\log y, 88,686-702$.

Keller, J. \& Bless, H. (2004). Evolutionary thought and psychological essentialism: The belief in genetic predisposition and its relationship to basic processes of social cognition. Journal of Cultural and Evolutionary Psychology, 2, 123-141.

Kelly, G. A. (1955). The psychology of personal constructs. New York: Norton.
Kluegel, J. R. \& Smith, E. R. (1986). Beliefs about inequality: Americans' view of what is and what ought to be. New York: Aldine De Gruyter.

Lerner, M. J. (1970). The desire for justice and reactions to victims. In J. M. Berkowitz (Ed.), Altruism and helping behavior (pp. 205-229). New York: Academic Press.

Levy, S. R., Chiu, C. Y. \& Hong, Y. Y. (2006). Lay theories and intergroup relations. Group Processes EO Intergroup Relations, 9, 5-24.

Levy, S. R., Freitas, A. L. \& Salovey, P. (2002). Construing action abstractly and blurring social distinctions: Implications for perceiving homogeneity among, but also empathizing with and helping, others. Journal of Personality and Social Psychology, 83, 1224-1238.

Levy, S. R., West, T., Ramírez, L. \& Karafantis, D. M. (2006). The protestant work ethic: A lay theory with dual intergroup implications. Group Processes \& Intergroup Relations, 9, 95-115.

Levy, S. R., West, T. \& Ramírez, L. (2005). Lay theories and intergroup relations: A social developmental perspective. The European Review of Social Psychology, 16, 189-220.

Leyens, J. P. Rodríguez-Pérez, A., Rodríguez-Torres, R., Gaunt, R., Paladino, P. M., Vaes, J. \& Demoulin, S. (2000). The emotional side of prejudice: The atribution of secondary emotions to ingroups and outgroups. Personality and Social Psychology Review, 4(2), 186-197.

McConahay, J. B. \& Hough, J. C. (1976). Symbolic racism. Journal of Social Issues, 32, 23-45.

Mahalingam, R. (2003). Essentialism, culture, and beliefs about gender among the Aravanis of Tamil Nadu, India. Sex Roles, 49, 489-496.

Mahalingam, R. \& Leu, J. (2005). Culture, essentialism, immigration, and representations of gender. Theory EO Psychology, 15, 839-860.

Mahalingam, R. \& Rodríguez, J. (2003) Essentialism, power and cultural Psychology of Gender. Journal of Cognition and Culture, 3(2), 157-174.

Medin, D. (1989) Concepts and conceptual structure. The American Psychologist, 44, 1469.

Medin, D. \& Ortony, A. (1989). Psychological essentialism. In S. Vosniadou \& A. Ortony (Eds.), 
Similarity and analogical reasoning (pp. 179-195). New York: Cambridge University Press.

Monteith, M. \& Walters, G. (1998, February). Egalitarianism, moral obligation, and prejudice-related. Personality \& Social Psychology Bulletin, 24(2), 186.

Niles, F. S. (1999). Toward a cross-cultural understanding of work-related beliefs. Human Relations, 52, 855-867.

Parales-Quenza, C. \& Vizcaíno-Gutiérrez, M. (2007). Las relaciones entre actitudes y representaciones sociales: elementos para una integración conceptual. Revista Latinoamericana de Psicología, 39(2), 351-361.

Pratto, F., Sidanius, J., Stallworth, L. M. \& Malle, B. F. (1994). Social dominance orientation: A personality variable predicting social and political attitudes. Journal of Personality and Social Psychology, 67, 741-763.

Rangel, U. \& Keller, J. (s.f.). Belief in social determinism as a component of psychological essentialism. Manuscrito no publicado.

Rothbart, M. \& Taylor, M. (1992). Category labels and social reality: Do we view social categories as natural kinds? In G. R. Semin \& K. Fiedler (Eds.), Language and Social Cognition (pp. 11-36). London: Sage.

Shaver, K. (1970). Defensive attribution: effects of severity and relevance on the responsibility assigned for an accident. Journal of Personality and Social Psychology, 14, 101-113.

Shaver, K. \& Drown, D. (1986). On causality, responsibility, and self blame: A theoretical note. Journal of Personality and Social Psychology, 50, 697-702.

Sidanius, J. \& Pratto, F. (1999). Social dominance: An intergroup theory of social hierarchy and oppression. New York: Cambridge University Press.

Sommerman, F. (1993). Value, attitude and belief determinants of willingness to accept a facility for the homeless. Journal of Social Distress and the Homeless, 2, 177-191.

Tajfel, H. (1984). Grupos Humanos y categorias sociales. Barcelona: Herder.
Tajfel, H., Flament, C., Billig, M. G. \& Bundy R. F. (1971). Social categorization and intergroup behavior. European Journal of Social Psychology, 1, 149-177.

Tajfel, H. \& Turner, J. C. (1979). An Integrative Theory of Intergroup Conflict. In W. G. Austin \& S. Worchel (Eds.), The Social Psychology of Intergroup Relations (pp. 94-109). Monterey, CA: Brooks-Cole.

Verkuyten, M. (2003). Discourses about ethnic group (de-) essentialism: Oppressive and progressive aspects. British Journal of Social Psychology, 42, 371-391.

Verkuyten, M. \& Brug, P. (2004). Multiculturalism and group status: The role of ethnic identification, group essentialism and protestant ethic. European Journal of Social Psychology, 34, 647-661.

Wegener, D. T. \& Petty, R. E. (1998). The naive scientist revisited: Naive theories and social judgment. Social Cognition, 16, 1-7.

Wyer, R. S., Jr., Bodenhausen, G. V. \& Gorman, T. F. (1985). Cognitive Mediators of Reactions to Rape. Journal of Personality and Social Psychology, 48(2), 324-338.

Williams, M. \& Eberhardt, J. (2008). Biological conceptions of race and the motivation to cross racial boundaries. Journal of Personality and Social Psychology, 94(6), 1033-1047.

Yzerbyt, V., Corneille, O. \& Estrada, C. (2001). The interplay of subjective essentialism and entitativity in the formation of stereotypes. Personality and Social Psychology Review, 5, 141-155.

Yzerbyt, V., Judd, C. M. \& Corneille, O. (2004). The psychology of group perception: Perceived variability, entitativity, and essentialism. New York: Psychology Press.

Yzerbyt, V., Rogier, A. \& Fiske, S. (1998). Group entitativity and social attribution: On translating situational constraints into stereotypes. Personality and Social Psychology Bulletin, 24, 1089-2015.

Zubieta, E. (2007). Creencias en la Ética Protestante del Trabajo (EPT) y competición en estudiantes universitarios. Perspectivas en Psicología, 4(1), 66-72. 